\title{
Uterine choriocarcinoma: A gynaecological masquerader case report and review of the literature
}

\author{
Eguzo Kelechi ${ }^{1}$, Chisara C. Umezurike ${ }^{1^{*}}$, Emmanuel Akwuruoha $^{2}$ \\ ${ }^{1}$ Nigerian Christian Hospital, Aba, Nigeria; ${ }^{*}$ Corresponding Author: umeztochi@hotmail.com \\ ${ }^{2}$ Abia State University Teaching Hospital, Aba, Nigeria
}

Received 9 July 2013; revised 15 August 2013; accepted 1 September 2013

Copyright (c) 2013 Eguzo Kelechi et al. This is an open access article distributed under the Creative Commons Attribution License, which permits unrestricted use, distribution, and reproduction in any medium, provided the original work is properly cited.

\begin{abstract}
Background: Choriocarcinoma is a rare clinical condition, and its diagnosis may be difficult, especially in resource-limited settings. Case Presentation: A 38-year old para 2 woman is with a 4-month history of intractable vaginal bleeding and offensive vaginal discharge, but without antecedent pregnancy. She had previously been managed at various tertiary medical institutions where several pelvic ultrasound scans and even histology of endometrial curette could not clinch the diagnosis. The diagnosis of choriocarcinoma was made by a serial strip-based pregnancy testing, which was still positive at 1:200 dilutions. She was treated with chemotherapy involving Adriamycin, Cyclophosphamide, Methotrexate and Folinic acid. Conclusion: The diagnosis of choriocarcinoma may be difficult especially when it develops ab initio without preceding abortion, molar or term pregnancy. In settings where serum hCG assay may be not available, the simple strip-based pregnancy test in dilution could be helpful in its diagnosis and treatment monitoring.
\end{abstract}

Keywords: Choriocarcinoma; Chemotherapy; Human Chorionic Gonadotropin; Resource-Limited Settings

\section{INTRODUCTION}

According to the World Health Organization Science Group in 1983 [1] on histopathological and clinical terminology, gestational choriocarcinoma is a carcinoma arising from the trophoblastic epithelium that shows both cytotrophoblastic and syncytiotrophoblastic element. It may arise from conceptions that give rise to a live birth, a still birth, and abortion at any stage, an ectopic pregnancy or a hydatidiform mole, or it may arise from $a b$ initio.

Gestational trophoblastic diseases (GTD) comprise a variety of biologically interrelated conditions which form a clinical spectrum consisting of four distinct clinical pathological entities namely, molar pregnancy, invasive mole, placental site trophoblastic tumours, and choriocarcinoma [2-4]. Serum human chorionic gonadotropin (h'CG) assay has remained the main stay of diagnosis, prognosis and monitoring of the condition.

The purpose of this report is to illustrate how difficult the diagnosis of choriocarcinoma could be and to show how a simple and "technology free" pregnancy test could still be relevant in its diagnosis and treatment in resourcepoor settings.

\section{CASE PRESENTATION}

A 38-year old para-2 Nigerian woman was presented in our centre with 4 months history of intractable vaginal bleeding. The bleeding was said to be heavy and was associated with passage of big blood clots. It was associated with offensive vaginal discharge, weight loss, fever and antecedent menorrhagia. It was not however associated with any history of amenorrhea, termination of pregnancy, lower abdominal swelling, post-coital or contact bleeding. Her last confinement was four years prior to presentation. There was no history of cough, hemoptysis, jaundice, unusual behaviour, convulsion, urinary or gastro-intestinal symptoms. Prior to her presentation at our center, the patient had been managed at the various tertiary hospitals (both in northern and south-eastern Nigeria), where various abdominal ultra-sound scan report suspected uterine fibroids, cervical cancer and endometrial cancer respectively. A histopathological report from one of the centres queried chronic endometritis. There was no definite diagnosis, but she had been transfused with many units of blood.

Initial evaluation revealed severe anaemia, with high grade fever, tachycardia (120 beats/minute) and low 
blood pressure (80/50 mmHg). Her chest was clinically clear, while she had suprapubic tenderness and offensive vaginal discharge associated with altered blood and cervical excitation tenderness. There was no suburethral or cervical lesion. Due to her previous conflicting investigation reports, the patient was booked for examination under anesthesia, where she had torrential bleeding that was coming from uterine cavity. Thus, a pregnancy test was ordered that led to the diagnosis of choriocarcinoma. Although a titrated serum hCG could not be done, an analysis of urine hCG was positive at 1:200 dilution. Her blood group was $\mathrm{B}^{+}$, while her husband's was $\mathrm{O}^{+}$. Her PCV on admission was $12 \%$ and her total white blood cell count, platelet count, serum electrolytes and urea were within normal limits.

She was successfully resuscitated with antibiotics and massive blood transfusion (20 units). Further evaluation did not show any evidence of lung, liver or brain metastases. Meanwhile, prognostic scoring of the patient was uncertain as she was not "pregnant" and the serum hCG could not be assayed, but she was placed at clinical stage I (tumour confined to the uterus). She was then commenced on cancer chemotherapy.

Therapy was instituted using Methotrexate $50 \mathrm{mg}$ on days 1, 3, 5 and 7; Folinic acid $7 \mathrm{mg}$ on days 2, 4, 6 and 8; Cyclophosphamide $650 \mathrm{mg}$ daily for 5 days (in saline), and Adriamycin $75 \mathrm{mg}$ stat on day 1 . Following the first course of chemotherapy, the bleeding ceased and the patient made tremendous clinical improvement. The course was repeated on day 21 , while the patient was consistently monitored for signs of toxicity. Pregnancy test became negative at the end of the second course. She was scheduled to receive one more course of chemotherapy but she defaulted due to high cost of the cytotoxic drugs and the apparent relief of her symptoms. She presented three months later with relapse and features of metastasis and died.

\section{DISCUSSION}

Gestational trophoblastic disease most commonly follows molar pregnancy and may also occur following normal or ectopic pregnancies and spontaneous or therapeutic abortions. Its incidence varies with figures as high as 1 in 120 pregnancies in some areas of Asia and South America, compared to 1 in 1200 in United States [5]. In the United Kingdom, it has a calculated incidence of 1 in 714 live births [3]. Metastatic gestational trophoblastic disease occurs in $4 \%$ patients after local management of Hydatidiform mole [5]. The incidence of choriocarcinoma after complete hydatidiform mole is about 1000 times greater than after a normal pregnancy . It may occur $a b$ initio as in the case presented [1,6]. Choriocarcinoma is a rare tumour. In western countries, the incidence is 1 in 45,000 to 50,000 pregnancies [5,7]. Higher incidence is reported from, Africa, Asia and South America [8]. Majority of cases occur in women aged less than 35 years of age [5].

Choriocarcinoma is suspected when there is persistent or irregular uterine haemorrhage, following abortion or hydatidiform mole. However, as illustrated in this case, it could also be suspected in cases of undiagnosed vaginal bleeding, even in the absence of pregnancy. Rapid growth and haemorrhage make the tumour a gynecological emergency. Metastasis may occur in lung, pelvis and vagina. Rare sites include gastrointestinal tract, spleen, and kidney. The central nervous system is seldom involved in the absence of pulmonary metastasis [2]. Metastatic disease occurs in $4 \%$ of patients after local management of hydatidiform mole and very rarely after term pregnancies or abortions [1]. Sometimes they may be combined with malignant germ cells components; and it may be difficult to differentiate a primary tumour from a metastasis. For females in non reproductive age group, the tumours may arise from ovarian germ cells, which is histologically similar to gestational uterine choriocarcinoma [6].

Hitherto, serum hCG assay has remained the key factor in diagnosis and monitoring of the progression of the disease. This has made clinical prognostic scoring easy and objective, as the serum hCG provides a sensitive and reliable indicator of the body burden of the tumour, its progression and regression in response to treatment [1]. However, in resource-limited settings, like ours, serial dilution test for urine hCG has also proven to be a useful diagnostic tool. The emphasis, however, should be to correlate the urine titers with serum values. This will enhance prognostication of patients for appropriate chemotherapy. According to I.A. McNeish et al. [9], proper classification of patients (as low risk) did not compromise outcome, but rather prevented undue exposure to potentially carcinogenic chemotherapy.

Traditionally, patients with uterine choriocarcinoma have been categorized as low risk (score of 0 to 5), medium risk (score of 6 to 9) and high risk (score $>9$ ), using the prognostic scoring system [1]. This enables rational use of chemotherapy with emphasis on the risk of developing resistance. However, I.A. McNeish et al. [9] have proposed a new scoring system: Charing Cross scoring system (Table 1), which is intended to further reduce the risk of undue exposure to chemotherapy. This new system proposes the use of Methotrexate-Folinic acid regimen for low risk disease (score 0 - 8), while EMA/CO (Etoposide, Methotrexate, Actinomycin D, Cyclophosphamide and Oncovin) is reserved for high risk disease (score $>8$ ). An algorithm (Figure 1) has also been developed to enhance case management.

Initial treatment for those with low-risk disease was Methotrexate (MTX) (50 mg intramuscularly on days 1, 


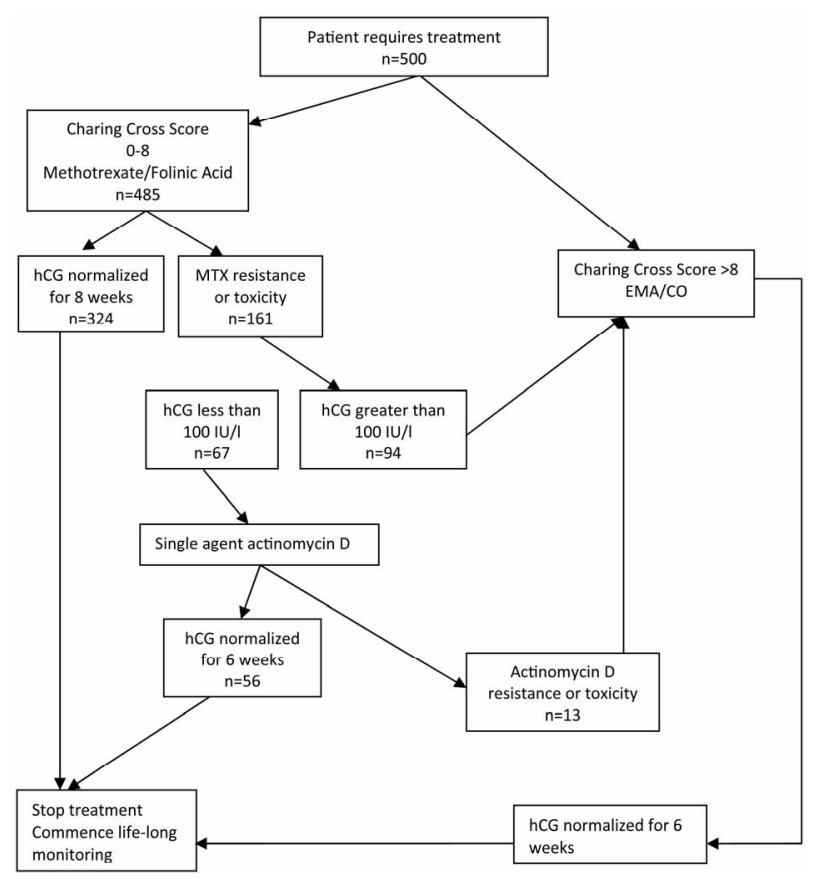

Figure 1. Treatment pathway for low-risk gestational trophoblastic disease.
3, 5, and 7 with Folinic acid (FA) 7.5 mg orally on days 2, 4, 6, and 8, repeated every 2 weeks. [1] Whereas for those with medium-risk disease, the chemotherapy regimen consisted of MTX as above, plus oral Hydroxyurea $1 \mathrm{~g}$ on day 1 , oral mercaptopurine $75 \mathrm{mg}$ on the same day as the FA, alternating with courses of either intravenous Etoposide $250 \mathrm{mg} / \mathrm{m}^{2}$ on days 1 and 3, or courses of intravenous Actinomycin $0.5 \mathrm{mg}$ daily for 5 days. 2 Treatment for high-risk disease was infusional chemotherapy according to the Etoposide, Methotrexate, and dActinomycin (EMA)/Cyclophosphamide and Vincristine (CO) regimen 3 (Table 2) [9].

Our case typically illustrates the frustration associated with cancer chemotherapy in Nigeria and indeed other developing countries where prevalent poverty makes procurement and sustenance of the drugs impossible.

Meanwhile, several complications of both uterine choriocarcinoma and its management have been documen ed. Being a highly malignant disease, metastases may occur in lung, pelvis and vagina. Rare sites include gastrointestinal tract, spleen, and kidney. The central nervous system is seldom involved in the absence of pulmonary metastasis [5]. There are reports describing

Table 1. Charing cross scoring system for gestational trophoblastic disease ${ }^{1}$.

\begin{tabular}{|c|c|c|c|c|}
\hline \multirow{2}{*}{ Variable } & \multicolumn{4}{|c|}{ Charing Cross Scoring System ${ }^{2}$} \\
\hline & 0 & 1 & 2 & 6 \\
\hline Age, years & $<39$ & $>39$ & & \\
\hline Antecedent pregnancy & Male & Abortion or unknown & Term & \\
\hline $\begin{array}{l}\text { Interval } \\
\text { (end of antecedent pregnancy to start treatment), months }\end{array}$ & $<4$ & $4-7$ & $6-12$ & $>12$ \\
\hline hCG, IU/L & $10^{3}-10^{4}$ & $<10^{3}$ & $10^{4}-10^{5}$ & $10^{5}$ \\
\hline \multirow[t]{3}{*}{ ABO (male $x$ female) } & & $\mathrm{A} \times \mathrm{O}$ & $\mathrm{B} \times \mathrm{A} / \mathrm{O}$ & \\
\hline & & $\mathrm{O} \times \mathrm{A}$ & $\mathrm{AB} \times \mathrm{A} / \mathrm{O}$ & \\
\hline & & O/A X unknown & & \\
\hline No. of metastases & 0 & $1-4$ & $4-8$ & $>8$ \\
\hline Site of metastases & Nil or lungs or vagina & Spleen, kidney & GI tract & CNS, liver \\
\hline Largest tumor mass, $\mathrm{cm}$ & $<3$ & $3-5$ & $>5$ & \\
\hline Previous chemotherapy & Nil & Nil & Single agent & Two or more drugs \\
\hline
\end{tabular}

${ }^{1}$ Low risk, 0 to 8 ; high risk, $>8 .{ }^{2}$ The patient's Charing Cross score is calculated at the start of treatment. All patients are assessed with clinical history, serum hCG, chest radiograph, and uterine ultrasound. If there is evidence of metastatic disease on radiograph, computed tomographic scan of the chest, abdomen and pelvis and a cranial magnetic imaging scan are obtained. The largest tumor mass measurement usually derives from the uterine ultrasound scan.

Table 2. Dactinomycin and EMA/CO chemotherapy regimens.

\begin{tabular}{|c|c|}
\hline Regimen & Dosage \\
\hline Dactinomycin & $0.5 \mathrm{mg}$ IV daily $\times 5$ Repeated every 2 weeks \\
\hline EMA/CO EMA Day 1 & $\begin{array}{l}\text { Dactinomycin } 0.5 \mathrm{mg} \text { IV bolus Etoposide } 100 \mathrm{mg} / \mathrm{m}^{2} \text { over } 30 \text { minutes } \\
\text { Methotrexate } 300 \mathrm{mg} / \mathrm{m}^{2} \text { over } 12 \text { hours }\end{array}$ \\
\hline Day 2 & $\begin{array}{l}\text { Dactinomycin } 0.5 \mathrm{mg} \text { IV bolus Etoposide } 100 \mathrm{mg} / \mathrm{m}^{2} \text { over } 30 \text { minutes } \\
\text { Folinic acid } 15 \mathrm{mg} \text { PO bid over } 2 \text { days commencing } 24 \text { hours after start of methotrexate }\end{array}$ \\
\hline CO & $\begin{array}{l}\text { Vincristine } 0.8 \mathrm{mg} / \mathrm{m}^{2} \text { (maximum } 2 \mathrm{mg} \text { ) bolus Cyclophosphamide } 600 \mathrm{mg} / \mathrm{m}^{2} \\
\text { over } 30 \text { minutes EMA and CO alternatively weekly }\end{array}$ \\
\hline
\end{tabular}


metastasis to large intestine from choriocarcinoma presenting as pseudo-obstruction and metastasis to stomach. Metastasis to gastrointestinal tract and presentation as perforation of small intestine is very rare [10]. Also, it has been reported that exposure to combination, but not single-agent, chemotherapy is associated with an increased incidence of second tumors, especially myeloid leukemia and colon cancer [11]. In addition, combination chemotherapy, more than single agent Methotrexate, increases the risk of an early menopause [12].

\section{CONCLUSION}

Although uterine choriocarcinoma, a rare form of gestational trophoblastic disease, usually follows a molar pregnancy, term deliveries or abortions, it can also present $a b$ initio. Its diagnosis can often pose a dilemma in resource-limited settings, but a high index of suspicion is helpful. The high cost of cytotoxin, which contributed to the default in treatment and eventual death of the patient, should be revisited. There is a need to make the cytotoxic drugs available and affordable across the world. Finally, although urine hCG assay is not the gold standard for the diagnosis and monitoring of this disease, it is useful in resource-limited settings. Further studies, however, should be done to correlate urine hCG titres with serum hCG levels in order to enhance the diagnosis and management of choriocarcinoma in low-resource settings.

\section{REFERENCES}

[1] James, F.H., et al. (1993) Cancer medicine. 3rd Ed, Lea \& Febinger, Philadelphia, 704, 1691-1694.

[2] Muggia, F.M., Eifel, P.J. and Burke, T.W. (1997) Gestational trophoblastic diseases. In: de Vita Jr., V.J., Hellman, S. and Rosenberg, S.A., Eds., Cancer Principles and Practice of Oncology, Lippincott-Raven, Philadelphia, 14271539.

[3] Royal College of Obstetricians and Gynaecologists (2010)
The management of gestational trophoblastic disease. RCOG, London.

[4] Ng, T.Y. and Wong, L.C. (2003) Diagnosis and management of gestational trophoblastic neoplasia. Best Practice \& Research Clinical Obstetrics and Gynaecology, 17, 893-903. http://dx.doi.org/10.1016/S1521-6934(03)00098-1

[5] Paradinas, F.J. (1992) Pathology and classification of trophoblastic tumours. In: Coppleson, M., Ed., Gynaecologic Oncology, 2nd Edition, Churchill Livingstone, London, 1013-1026.

[6] Acosta-Sison, H. (1959) Ab initio choriocarcinoma: Two unusual cases. Obstetrics \& Gynecology (New York), 13, 350-352.

[7] Seckl, M.J., Sabire, N.J. and Berkowitz, R.S. (2010) Gestational trophoblastic disease. The Lancet, 376, 717-729. http://dx.doi.org/10.1016/S0140-6736(10)60280-2

[8] Fox, H. and Buckley, C.H. (1992) The female genital tract and ovaries. In: McGee, J.O.D., Isaacson, P.G. and Wright, N.A., Eds., Oxford Text Book of Pathology, 2nd Edition, Oxford University Press, New York, 1565-1639.

[9] Balagopal, P.G., et al. (2003) Unusual presentation of choriocarcinoma. World Journal of Surgical Oncology, 2003, 1, 4. http://dx.doi.org/10.1186/1477-7819-1-4

[10] McNeish, I.A., et al. (2002) Low-risk persistent gestational trophoblastic disease: Outcome after initial treatment with low-dose methotrexate and folinic acid from 1992 to 2000. Journal of Clinical Oncology, 20, 18381844. http://dx.doi.org/10.1200/JCO.2002.07.166

[11] Rustin, G.J.S., Newlands, E.S., Lutz, J.M., et al. (1996) Combination but not single-agent Methotrexate chemotherapy for gestational trophoblastic tumors increases the incidence of second tumors. Journal of Clinical Oncology, 14, 2769-2773.

[12] Bower, M., Rustin, G.J.S., Newlands, E.S., et al. (1998) Chemotherapy for gestational trophoblastic tumours hastens menopause by 3 years. European Journal of Cancer, 34, 1204-1207. http://dx.doi.org/10.1016/S0959-8049(98)00059-8 